\title{
Progression of field loss after trabeculectomy: a five-year follow-up
}

\author{
MARTIN N KIDD AND MARTIN O'CONNOR \\ From the Glaucoma Unit, Belfast City Hospital, Belfast BT9 7AB, Northern Ireland
}

SUMMARY We report a retrospective study of 60 eyes from 46 patients all with primary open-angle glaucoma who had a trabeculectomy to one or both eyes. A minimum five-year follow-up was available. The percentage pressure reductions at the one and five years postoperatively is shown. In a subgroup of 50 eyes which had Goldmann fields documented at least six-monthly for a minimum of five years postoperatively there was a continuation of glaucomatous field loss in $18 \%$ of eyes despite postoperative pressures in the normal range. The five-year percentage pressure reduction and the minimum percentage pressure reductions were statistically significant in a comparison between the field loss and no field loss groups. However, in the individual case there seemed no way of predicting which eyes would develop postoperative field loss, and regular postoperative perimetric assessment was found to be essential whatever the pressure measurements recorded.

Trabeculectomy is now generally considered to be a useful and relatively safe procedure in the management of chronic open-angle glaucoma. There is a trend towards earlier surgical intervention in the disease without necessarily establishing failure of 'maximum' medical therapy but proceeding instead to surgery if one or two medical regimens fail to reduce the pressure to satisfactory levels. Watson and Grierson' and others have stated that early surgical intervention in most cases is desirable and that long-term topical treatment has undesirable side effects (particularly with those agents causing an under-perfusion of the meshwork). However, we emphasise that despite apparently good postoperative control, in some patients, field loss continues to occur. Although there are many reports of the results of trabeculectomy and other pressure-lowering operations, ${ }^{2-5}$ relatively few of them report on a minimum five-year follow-up, particularly with regard to continued field loss.

\section{Subjects and methods}

This retrospective study included 46 patients, 16 male and 30 female, aged between 39 and 79 years at the time of surgery, with a mean age of 61 years. All

Correspondence to Martin N Kidd, FRCS, Department of Ophthalmology, Royal Victoria Hospital, Belfast BT12 6BA, Northern Ireland. patients were diagnosed as having primary openangle glaucoma on the basis of raised intraocular pressures (greater than $21 \mathrm{mmHg}$ ), glaucomatous field defects, and open angles.

The intraocular pressure in a total of 60 eyes from the 46 patients was uncontrolled on maximum medical therapy (two topical agents plus or minus a carbonic anhydrase inhibitor).

The 60 eyes included in the study had had only one drainage procedure (trabeculectomy) and were followed up for a minimum of five years postoperatively. In all cases the standard Watson modification ${ }^{6}$ of the Cairns procedure ${ }^{7}$ was performed.

All the patients were followed up at the Glaucoma Unit of the Belfast City Hospital. In 50 of the eyes Goldmann perimetry was performed at six-monthly intervals from between six months and six years preoperatively and at least six-monthly intervals postoperatively for a minimum of five years. All fields were tested by the same associate specialist at the unit. The field charts were examined by one of us (MNK) in a manner ensuring that the date of the operation was unknown. Progression of glaucomatous field loss was juidged to have occurred when one or more of the following criterias were met: (1) widening of a nasal step or other field defect by $5^{\circ}$ or more; (2) the appearance of a new scotomatous defect; (3) a change for relative to absolute scotoma.

Intraocular pressures were estimated by applanation tonometry. The mean of the preoperative read- 
Table 1 Intraocular pressures with and without treatment (TR) at the end of the one-and five-year postoperative periods

\begin{tabular}{llllll}
\hline Pressure & \multicolumn{2}{l}{ I Year postop. } & & \multicolumn{2}{c}{5 Years postop. } \\
\cline { 2 - 3 } \cline { 6 - 6 } & No. & $\%$ & & No. & $\%$ \\
\hline$<21$ (No TR) & 52 & $86 \cdot 6$ & & 45 & $75 \cdot 0$ \\
$\leqslant 21$ (With TR) & 2 & $3 \cdot 33$ & & 12 & $20 \cdot 0$ \\
$>21$ (With TR) & 6 & $10 \cdot 0$ & & 3 & $5 \cdot 0$ \\
\hline
\end{tabular}

ings for each eye was noted, as were the mean values of the intraocular pressures taken during the one- to five-year postoperative periods.

Data on the eyes from the groups with and without field loss were subjected to statistical analysis. The data were non-parametric, and the Mann-Whitney test was used throughout.

\section{Results}

Forty-five eyes $(75 \%)$ required no postoperative medication throughout the study period. Fifty-two eyes were receiving no medication at the end of the first postoperative year, and, of these, seven $(13.46 \%)$ required medication at the end of the fiveyear period (Table 1 ). The mean preoperative intraocular pressure of the 60 eyes was $25.1 \mathrm{mmHg}$. The mean postoperative intraocular pressure at the first visit was $13.04 \mathrm{mmHg}$. The mean of all the postoperative intraocular pressures at the end of the first year (with or without treatment) was $13.76 \mathrm{mmHg}$, and the mean of the postoperative intraocular pressures at the end of five years (with or without treatment) was $14.95 \mathrm{mmHg}$.

Table 1 shows that six eyes had a pressure in excess

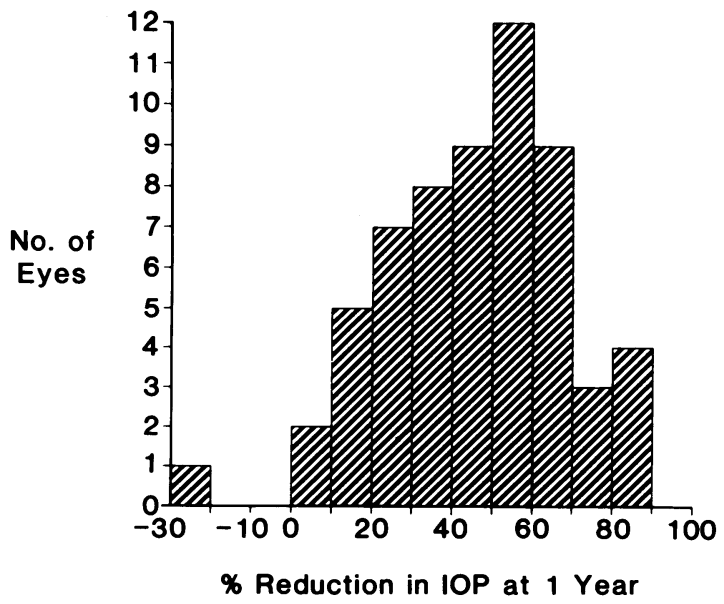

Fig. 1a Percentage reduction in IOP at end of one-year period.
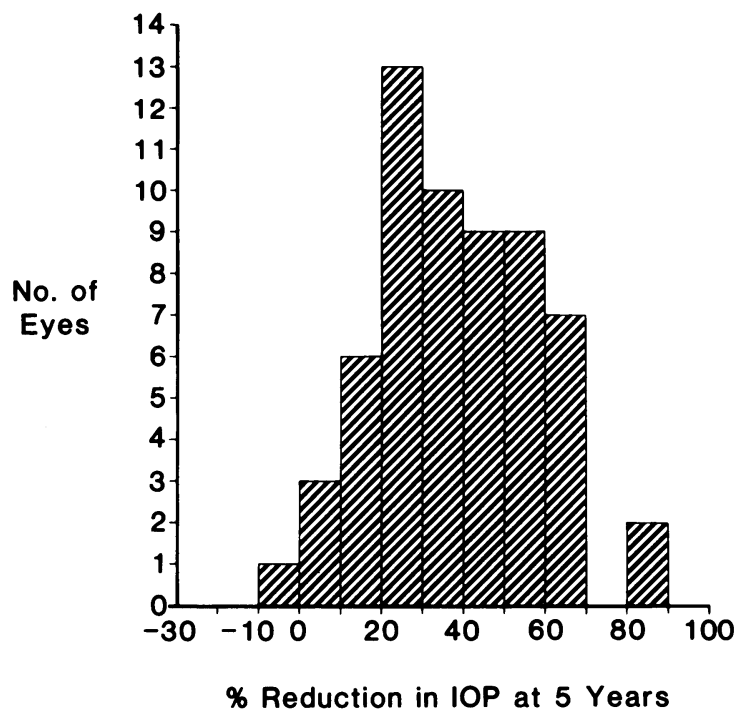

Fig. $1 \mathrm{~b}$ Percentage reduction in IOP at end of five-year period.
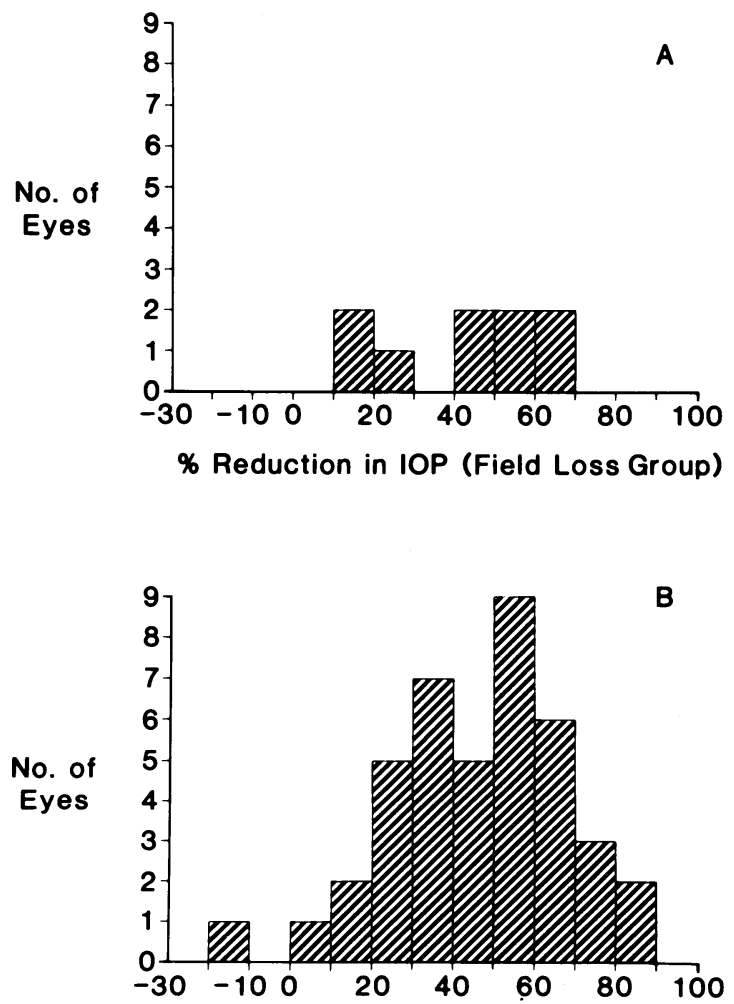

\% Reduction in IOP (No Field Loss Group)

Fig. 2 Percentage reduction in IOP at end of one-year postoperative period for the 'field loss' (A) and 'no field loss' (B) groups. 

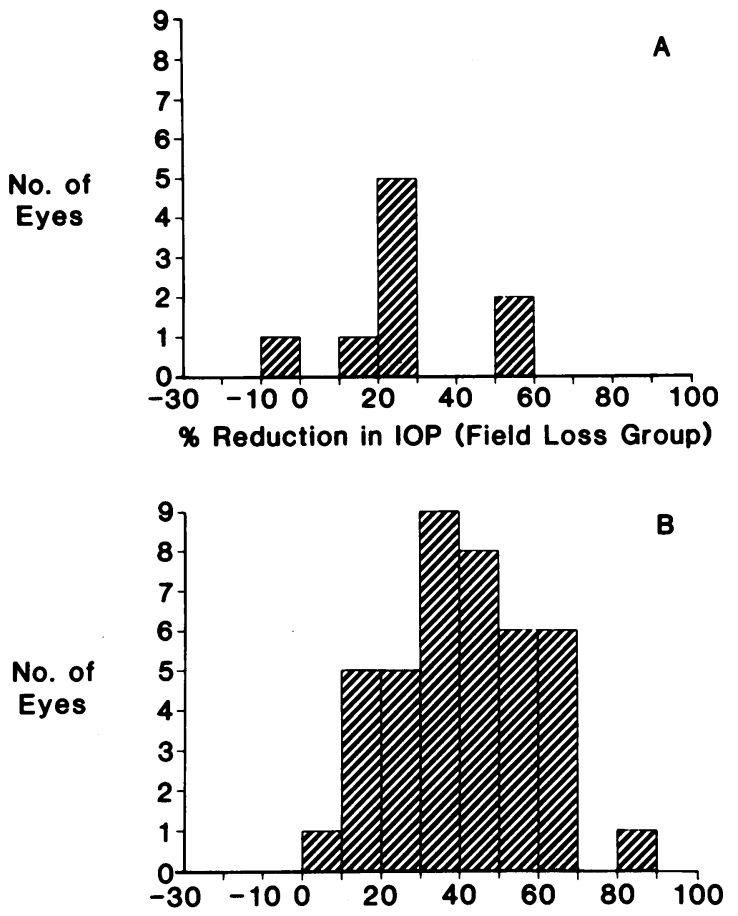

\% Reduction in IOP (No Field Loss Group)

Fig. 3 Percentage reduction in IOP at end of five-year postoperative period for the 'field loss' (A) and 'no field loss' (B) groups.

of $21 \mathrm{mmHg}$ with medication at the end of the first postoperative year, and the figure for this category at the end of the five-year period was three eyes. Three eyes had additional medical therapy which brought them down to less than $21 \mathrm{mmHg}$.

Figs. 1a and 1b show histogramatically the percentage pressure reduction at the end of the one- and five-year periods of study with or without therapy. There was a slight trend towards less reduction in pressure when the one- and five-year periods are compared. The mean of the percentage reduction in
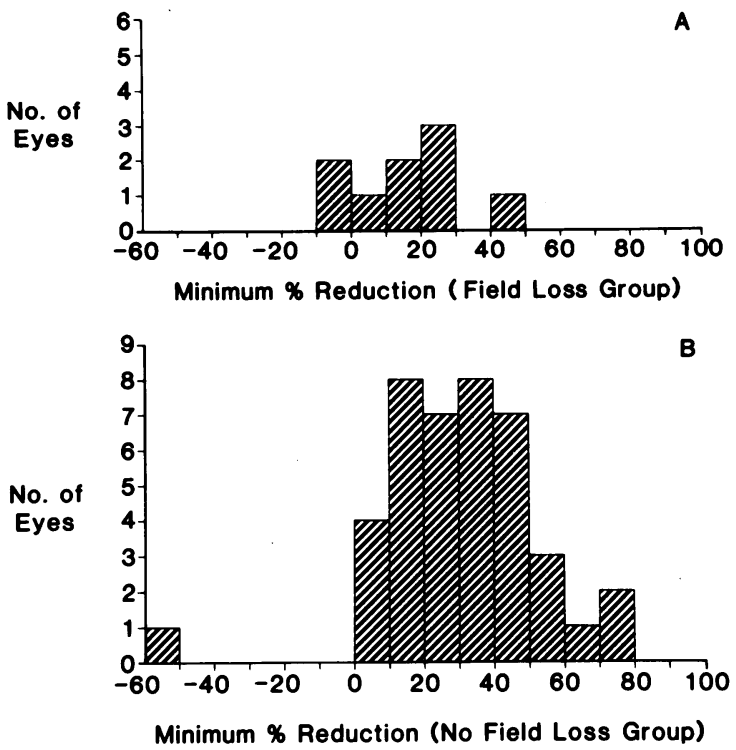

Fig. 4 Minimum percentage reduction in IOP throughout the study period for the 'field loss' (A) and 'no field loss' (B) groups.

pressure at the end of the one-year period was $45.26 \%$, and the mean percentage reduction at the end of the five-year period was $39 \cdot 23 \%$.

In the subgroup of 50 eyes for which field documentation over the period of study was available nine $(18.0 \%)$ had glaucomatous field loss at the end of the fifth postoperative year. Figs. 2 and 3 show the percentage pressure reductions at one and five years respectively for the 'progression of field loss' and 'no progression of field loss' groups.

Fig. 4 shows the minimum percentage pressure reductions in the 'progression of field loss' and 'no progression of field loss' groups. This is calculated from the highest recorded postoperative intraocular pressure reading.

Although a trend is seen towards less percentage pressure reduction (Table 2) at the end of the first

Table 2 The various mean values for the 'field loss' and 'no field loss' groups, showing the significant values

\begin{tabular}{|c|c|c|c|}
\hline & Progression of field loss group & No progression of field loss group & $p$ \\
\hline No. of eyes & 9 & 41 & \\
\hline Percentage & $18 \cdot 0$ & $82 \cdot 0$ & \\
\hline Mean 1 year postop. IOP & $14 \cdot 22$ & $13 \cdot 92$ & 0.48 \\
\hline Mean 5 ycars postop. IOP & $17 \cdot 33$ & 14.92 & $0 \cdot 06$ \\
\hline Mean of minimum \% reduction & $15 \cdot 56$ & $29 \cdot 72$ & $0.03^{*}$ \\
\hline Mean 1 year $\%$ reduction & $34 \cdot 5$ & $44 \cdot 2$ & 0.48 \\
\hline Mean 5 years $\%$ reduction & $26 \cdot 6$ & $40 \cdot 34$ & $0 \cdot 03^{*}$ \\
\hline Mean of age at date of op. (years) & $62 \cdot 1$ & $61 \cdot 1$ & \\
\hline
\end{tabular}

*Significant at $5 \%$ level. 
year postoperatively between the two field groups, it was only at the end of the five-year period that the trend is significant. The mean of the minimum percentage pressure reduction in the two groups is significant at the $5 \%$ level.

Fig. 5 shows the immediate preoperative and fouryear postoperative Goldmann fields of a female patient of 58 years at the time of surgery. Her recorded postoperative pressures were never above $17 \mathrm{mmHg}$. It can be seen that glaucomatous field loss progressed.

Fig. 6 is another example of a patient in the 'progression of field loss' group. This female was 61 years of age at the time of surgery. Her postoperative pressures were never recorded above $12 \mathrm{mmHg}$. The figure shows progression of the field when the oneyear and four-year postoperative fields are compared. No preoperative disc photographs were available for study when comparing the "progression of field loss' and 'no progression of field loss' groups, but on examining the presurgical fields from the two groups we found a similar distribution in each group of early, moderate, and late glaucomatous field loss according to the criteria outlined.

Only two of the nine eyes in the 'progression of field loss' group had extensive initial field loss. The other seven showed early field loss represented by only a small arcuate scotoma. Fig. 6 is typical. It was decided to ignore other observers' comments in the medical charts on the state of optic disc cupping.
Fig. 5 Immediate preoperative and four-year postoperative Goldmann fields of a patient in the 'field loss' group (see text).
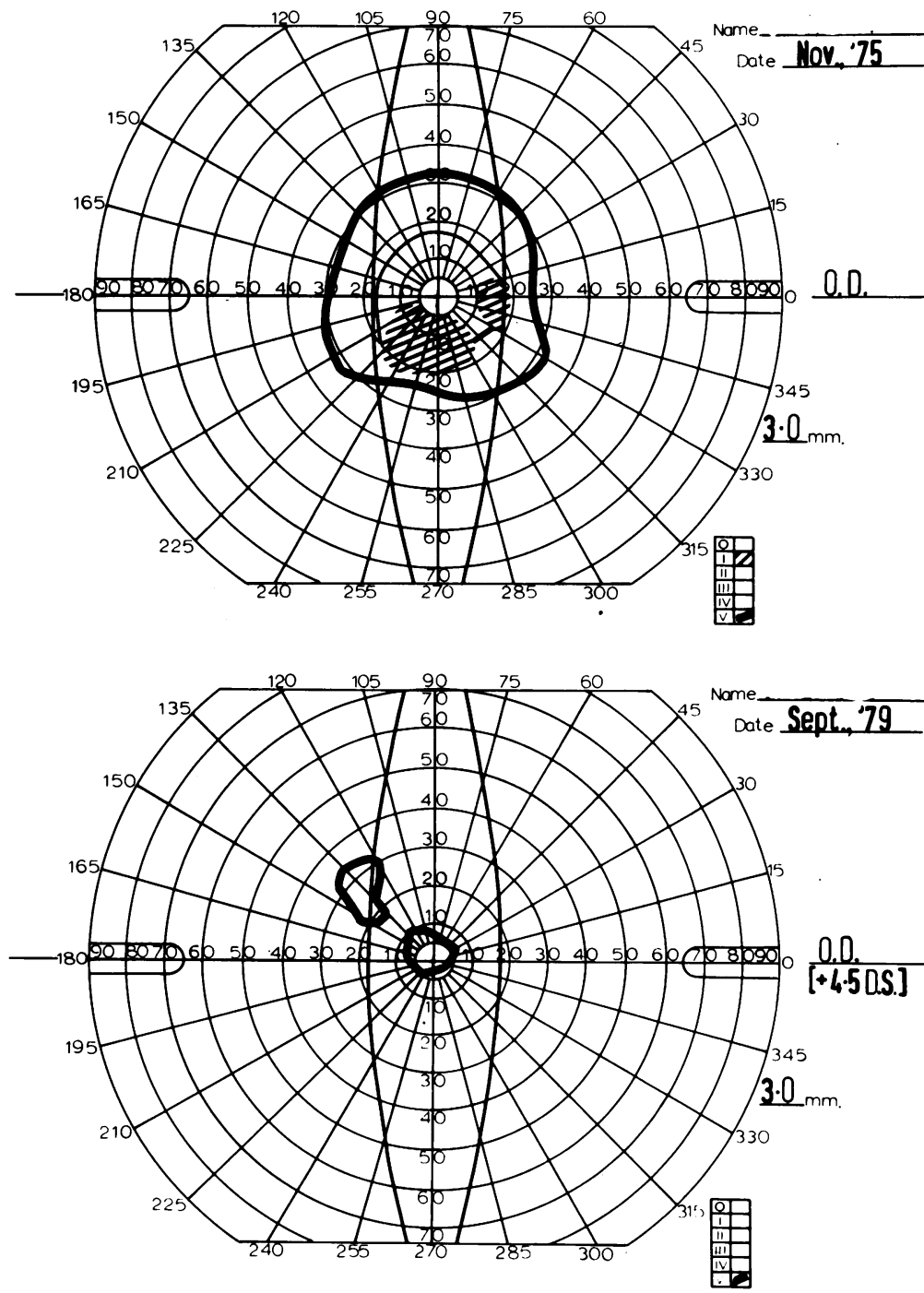

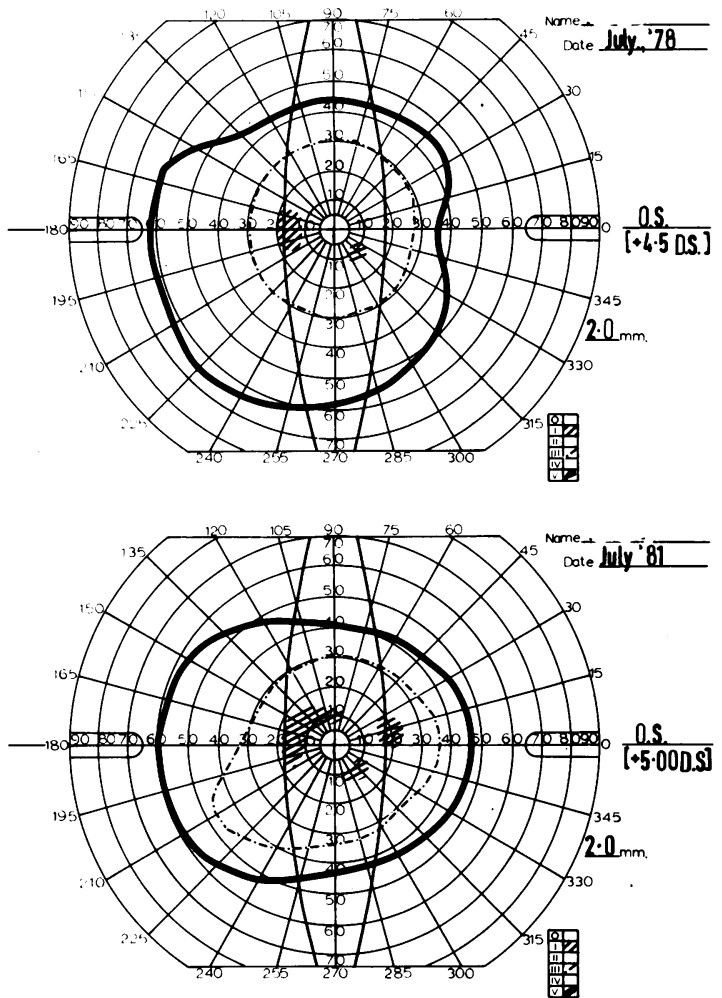

Fig. 6 One-and four-year postoperative Goldmann fields of a patient in the 'field loss' group (see text).

\section{Discussion}

Although it is becoming increasingly apparent that there is no simple correlation between raised intraocular pressure and progression of field loss and that other factors may play a part, it is still common practice to consider chronic open-angle glaucoma 'cured' when postoperative intraocular pressures remain within normal limits.

Other factors which may be important are age, generalised microvascular disease, systemic hypertension, and genetic factors.

In this study we noted that nine out of 50 eyes (18\%) continued to lose field postoperatively despite satisfactory pressure measurements. Rollins and Drance $^{4}$ cite a figure of $29 \%$ progression of field loss despite pressure measurements postoperatively in the normal range.

Although the mean one- and five-year postoperative readings of intraocular pressure for the "no progression of field loss' group were less than those of the 'progression of field loss' group, the difference was not significant at the $\mathbf{5 \%}$ level. Statistical significance was found, however, at this level for the mean of the minimum percentage reduction for the 'progression of field loss' and 'no progression of field loss' groups. This indicates that in the 'no progression of field loss' group there was less variation in individual pressure measurements.

In addition the difference in the mean five-year percentage reduction in intraocular pressures between the two groups was significant at the $5 \%$ level. Although a similar trend is shown at the end of one year after operation, it was not shown to be significant.

The results are in agreement with the findings of others $^{45}$ in showing that the reduction of the intraocular pressure by surgical means does not necessarily protect the eye from further field loss. Surgery, however, may reduce the rate of field loss in every patient. Although we did not have accurate documentation of disc changes in our patients, we recognise the susceptibility of the severely cupped disc to nerve fibre loss at normal intraocular pressures. However, we noted that the distribution of early, moderate, and late field loss, and therefore presumably related nerve fibre loss, was similar in the groups under consideration.

The difference between the two field groups in the minimum percentage reduction and the five-year percentage reduction in intraocular pressure was statistically significant, but in the individual patient's case there seems no way of forseeing this, and we emphasise the need for regular perimetry after trabeculectomy regardless of the intraocular measurements. If further field loss is noted, accurate phasing should be undertaken and consideration given to a further reduction in the IOP by medical means.

Grateful thanks are extended to Dr Doreen Linton of the Glaucoma Unit, who performed the perimetry in the study.

\section{References}

1 Watson PG, Grierson I. The place of trabeculectomy in the treatment of glaucoma. Ophthalmology (Rochester) 1981; 88: 175-95.

2 Werner EB, Drance SM. Progression of glaucomatous ficld change despitc successful filtration. Can J Ophthalmol 1977; 12: 275-80

3 Greve EL, Dake L. Four year follow-up of a glaucoma operation. Int Ophthalmol 1979; 1: 139-45.

4 Rollins DF, Drance SM. Five year follow-up of trabeculectomy in the management of chronic open angle glaucoma. Symposium on glaucoma. Trans New Orleans Acad Ophthalmol. St Louis: Mosby, 1981: 295-30).

5 Werner EB, Drance SM, Schulzer M. Trabeculectomy and the progression of glaucomatous ficld loss. Arch Ophthalmol 1977; 95: $1376-7$

6 Watson PG. Trabeculectomy: a modificd ab externo technique. Ann Ophthalmol 1970; 2: 199-205.

7 Cairns JE. Trabcculectomy-preliminary report of a new method. Am J Ophthalmol 1968; 66: 673-9. 